\title{
Impact Parameter Dependent Parton Distributions for a Relativistic Composite System
}

\author{
D. Chakrabarti* \\ Department of Physics, University of Florida, Gainesville, FL-32611-8440, USA
}

A. Mukherjeet

Institute Lorentz, University of Leiden, 2300 RA Leiden, Netherlands

(Dated: November 26, 2018)

\begin{abstract}
We investigate the impact parameter dependent parton distributions for a relativistic composite system in light-front framework. We take an effective two-body spin- $1 / 2$ state, namely an electron dressed with a photon in QED. We express the impact parameter dependent parton distributions in terms of overlaps of light-cone wave functions. We obtain the scale dependence of both fermion and gauge boson distributions and show the distortion of the pdfs in the transverse space for transverse polarization of the state at one loop level.
\end{abstract}

*Electronic address: dipankar@phys.ufl.edu

$\dagger$ Electronic address: asmita@lorentz.leidenuniv.nl 


\section{INTRODUCTION}

Impact parameter dependent parton distributions $q\left(x, b^{\perp}\right)[1,2]$ have been introduced recently as a physical interpretation of generalized parton distributions (GPDs) 3] in terms of probability densities in the impact parameter space. It is known that GPDs are off-forward matrix elements of light-cone bilocal operators and they do no have a probabilistic interpretation like ordinary parton distributions. $q\left(x, b^{\perp}\right)$ can be expressed as Fourier transform of GPDs with respect to the transverse momentum transfer (when the longitudinal momentum transfer is zero) and they give the distribution of partons in the transverse position space. In fact they obey certain positivity constraints and thus it is legitimate to associate the physical interpretation as a probability density. This interpretation is not limited by relativistic effects in the infinite momentum frame. Another interesting aspect is that when the state is polarized in the transverse direction, the unpolarized impact parameter dependent pdf is distorted in the transverse plane [1]. This distortion can be linked to the transverse single spin asymmetries observed in transversely polarized Lambda production in $p p$ or $p \bar{p}$ collisions. Recently it has been shown in the framework of the scalar diquark model that the impact parameter space asymmetry together with the final state interaction of the active quark gives rise to the Sivers effect [4] in momentum space [5].

Impact parameter dependent pdfs have been investigated in chiral quark model for the pion [6], in the constituent quark model [7], in terms of a power-law ansatz of the lightcone wave function for the pion [8], in the context of investigating the color transparency phenomena [9], in the transverse lattice framework for the pion [10] and on the lattice [11]. In this work, we investigate both fermion and gauge boson distributions for a relativistic composite system in the light-front framework, taking into account the correlation between different Fock components of the light-cone (or light-front) wave function in light-front gauge. It is well known that the light-front formalism is especially suitable to investigate many aspects of relativistic bound states because of the Galilean transverse boost operators and the triviality of the vacuum [12] when a cutoff is imposed on the longitudinal momentum.

We take an effective spin $\frac{1}{2}$ system, namely an electron [13], dressed with a photon in QED. It is known that such a model is self consistent and has been used to investigate the helicity structure of a composite relativistic system [14]. The state can be expanded in Fock space in terms of light-cone wave functions. The light-cone wave functions in this case can be 
obtained from perturbation theory, and thus their correlations are known at a certain order in the coupling constant. However, their general form provides a template for parametrizing the more realistic composite system, namely the hadronic wave function 14]. Instead of an electron, one can also consider a state like a dressed quark in perturbative QCD. In fact this can be thought of as a field theoretic parton model where the partons, or the quarks and gluons, have mass, intrinsic transverse momenta and they interact. Previous studies have shown that this gives an intuitive picture of the DIS structure functions and scaling violations 15] and is suitable to address issues related to the spin and orbital angular momentum of the nucleon [16, 17]. Such a state has also been used to investigate the twist three GPDs in terms of overlaps of light-cone wave functions [18]. The aim of the present work is to extend these studies to the impact parameter dependent fermion and gauge boson pdfs in order to obtain a qualitative behavior in the impact parameter space within the framework of perturbation theory.

The plan of the paper is as follows. In section II, we give the definition of the impact parameter dependent pdf, In sections III and IV we calculate the fermion and gauge boson distributions respectively. Summary and conclusions are given in section V.

\section{IMPACT PARAMETER DEPENDENT PARTON DISTRIBUTIONS} 1]:

Impact parameter dependent pdfs are defined by considering a transversely localized state

$$
\left|P^{+}, R^{\perp}=0, \lambda\right\rangle=N \int \frac{d^{2} P^{\perp}}{(2 \pi)^{2}}\left|P^{+}, P^{\perp}, \lambda\right\rangle,
$$

where $\left|P^{+}, P^{\perp}, \lambda\right\rangle$ is the light cone helicity eigenstate with momentum $P$ and helicity $\lambda$, $N$ is a normalization factor. For a state with total momentum $P^{+}$the transverse center of momentum $R^{\perp}$ is defined as,

$$
R^{\perp}=\frac{1}{2 P^{+}} \int d x^{-} d^{2} x^{\perp} \Theta^{++} x^{\perp},
$$

where $\Theta^{\mu \nu}$ is the energy-momentum tensor.

The states $\left|P^{+}, P^{\perp}, \lambda\right\rangle$ can be thought of as helicity eigenstates in the infinite momentum

frame [1]. Due to the fact that the light-front transverse boost $B^{\perp}$ behaves similar to the non-relativistic Galilean Boost generators, it can be shown that the states $\left|P^{+}, R^{\perp}=0, \lambda\right\rangle$ 
defined above are simultaneous eigenstates of the longitudinal light cone momentum $P^{+}$, transverse position operator $R^{\perp}$ and light cone helicity $J^{z}$. The transverse position operator $R^{\perp}$ in fact is related to $B^{\perp}: R^{\perp}=-\frac{1}{P^{+}} B^{\perp}$.

The impact parameter dependent pdf is defined as,

$$
q\left(x, b^{\perp}\right)=\left\langle P^{+}, R^{\perp}=0^{\perp}, \lambda\left|O_{q}\left(x, b^{\perp}\right)\right| P^{+}, R^{\perp}=0^{\perp}, \lambda\right\rangle
$$

with

$$
O_{q}\left(x, b^{\perp}\right)=\int \frac{d y^{-}}{4 \pi} \bar{\psi}\left(-\frac{y^{-}}{2}, b^{\perp}\right) \gamma^{+} \psi\left(\frac{y^{-}}{2}, b^{\perp}\right) e^{\frac{i}{2} x P^{+} y^{-}}
$$

$b^{\perp}$ is the impact parameter, which is the transverse distance of the active quark from the center of mass. We have taken the light-front gauge, $A^{+}=0$. Instead of the fermion operator, one can also have a gauge boson operator

$$
O_{g}\left(x, b^{\perp}\right)=\int \frac{d y^{-}}{4 \pi} F^{+\nu}\left(-\frac{y^{-}}{2}, b^{\perp}\right) F^{+}{ }_{\nu}\left(\frac{y^{-}}{2}, b^{\perp}\right) e^{\frac{i}{2} x P^{+} y^{-}}
$$

to define the gauge boson pdf $g\left(x, b^{\perp}\right)$ similar to Eq. (2.3).

It can be shown that $q\left(x, b^{\perp}\right)$ can be expressed as a Fourier transform of the GPD $H_{q}\left(x, 0, \Delta^{2}\right)[1]:$

$$
q\left(x, b^{\perp}\right)=\mathcal{H}_{q}\left(x, b^{\perp}\right)=\int \frac{d^{2} \Delta^{\perp}}{(2 \pi)^{2}} e^{-i b^{\perp} . \Delta^{\perp}} H_{q}\left(x, 0, \Delta^{2}\right) .
$$

where $\Delta^{2}$ is the total momentum transfer and it is purely transverse. One can obtain a similar relation for the gauge boson counterpart.

\section{FERMION DISTRIBUTIONS}

We first calculate the off-forward matrix elements, parametrized in terms of the twist-two GPSs $H_{q}\left(x, \Delta^{2}\right)$ and $E_{q}\left(x, \Delta^{2}\right)$ in the standard way [20] and using the light cone spinors 21]. We have,

$$
\int \frac{d z^{-}}{8 \pi} e^{\frac{i}{2} x \bar{P}^{+} z^{-}}\left\langle P^{\prime} \uparrow\left|\bar{\psi}\left(-\frac{z^{-}}{2}\right) \gamma^{+} \psi\left(\frac{z^{-}}{2}\right)\right| P \uparrow\right\rangle=H_{q}\left(x, \Delta^{2}\right),
$$

where $H\left(x, \Delta^{2}\right)=H\left(x, 0, \Delta^{2}\right)$. The momentum of the initial state is $P^{\mu}$ and that of the final state is $P^{\prime \mu}$. The average momentum between initial and final state is then $\bar{P}^{\mu}=\frac{P^{\mu}+P^{\prime \mu}}{2}$. The momentum transfer is given by $\Delta^{\mu}=P^{\prime \mu}-P^{\mu}, P_{\perp}^{\prime}=-P_{\perp}=\frac{\Delta_{\perp}}{2}$. We take the 
skewedness $\xi=0$, in other words, the momentum transfer is purely transverse. We exclude the impact parameter space representation of GPDs for non-zero skewedness [19] from our analysis here.

We take the state $|P, \sigma\rangle$ to be a dressed electron consisting of bare states of an electron and an electron plus a photon :

$$
\begin{aligned}
|P, \sigma\rangle= & \mathcal{N}\left[b^{\dagger}(P, \sigma)|0\rangle\right. \\
+ & \sum_{\sigma_{1}, \lambda_{2}} \int \frac{d k_{1}^{+} d^{2} k_{1}^{\perp}}{\sqrt{2(2 \pi)^{3} k_{1}^{+}}} \int \frac{d k_{2}^{+} d^{2} k_{2}^{\perp}}{\sqrt{2(2 \pi)^{3} k_{2}^{+}}} \sqrt{2(2 \pi)^{3} P^{+}} \delta^{3}\left(P-k_{1}-k_{2}\right) \\
& \left.\phi_{2}\left(P, \sigma \mid k_{1}, \sigma_{1} ; k_{2}, \lambda_{2}\right) b^{\dagger}\left(k_{1}, \sigma_{1}\right) a^{\dagger}\left(k_{2}, \lambda_{2}\right)|0\rangle\right] .
\end{aligned}
$$

Here $a^{\dagger}$ and $b^{\dagger}$ are bare photon and electron creation operators respectively and $\phi_{2}$ is the multiparton wave function. It is the probability amplitude to find one electron plus photon inside the dressed electron state.

We introduce Jacobi momenta $x_{i}, q_{i}{ }^{\perp}$ such that $\sum_{i} x_{i}=1$ and $\sum_{i} q_{i}{ }^{\perp}=0$. They are defined as

$$
x_{i}=\frac{k_{i}^{+}}{P^{+}}, \quad q_{i}^{\perp}=k_{i}^{\perp}-x_{i} P^{\perp} .
$$

Also, we introduce the wave function,

$$
\psi_{2}\left(x_{i}, q_{i}^{\perp}\right)=\sqrt{P^{+}} \phi_{2}\left(k_{i}^{+}, k_{i}^{\perp}\right)
$$

which is independent of the total transverse momentum $P^{\perp}$ of the state and boost invariant. The state is normalized as,

$$
\left\langle P^{\prime}, \lambda^{\prime} \mid P, \lambda\right\rangle=2(2 \pi)^{3} P^{+} \delta_{\lambda, \lambda^{\prime}} \delta\left(P^{+}-P^{\prime+}\right) \delta^{2}\left(P^{\perp}-P^{\prime \perp}\right) .
$$

The two particle wave function depends on the helicities of the electron and photon. Using the eigenvalue equation for the light-cone Hamiltonian, this can be written as [15],

$$
\begin{gathered}
\psi_{2 \sigma_{1}, \lambda}^{\sigma}\left(x, q^{\perp}\right)=-\frac{x(1-x)}{\left(q^{\perp}\right)^{2}+m^{2}(1-x)^{2}} \frac{1}{\sqrt{(1-x)}} \frac{e}{\sqrt{2(2 \pi)^{3}}} \chi_{\sigma_{1}}^{\dagger}\left[2 \frac{q^{\perp}}{1-x}+\frac{\tilde{\sigma}^{\perp} \cdot q^{\perp}}{x} \tilde{\sigma}^{\perp}\right. \\
\left.-i m \tilde{\sigma}^{\perp} \frac{(1-x)}{x}\right] \chi_{\sigma} \epsilon_{\lambda}^{\perp *} .
\end{gathered}
$$

$m$ is the bare mass of the electron, $\tilde{\sigma}^{2}=-\sigma^{1}$ and $\tilde{\sigma}^{1}=\sigma^{2}$. In our case, $\Delta^{2}=-\left(\Delta^{\perp}\right)^{2}$ as $\xi=0 . \mathcal{N}$ gives the normalization of the state [15]:

$$
\mathcal{N}^{2}=1-\frac{\alpha}{2 \pi} \int_{\epsilon}^{1-\epsilon} d x\left[\frac{1+x^{2}}{1-x} \log \frac{Q^{2}}{\mu^{2}}-\frac{1+x^{2}}{1-x}+(1-x)\right],
$$


within order $\alpha$. Here $\epsilon$ is a small cutoff on $x$. We have taken the cutoff of the transverse momenta to be $Q^{2}, \mu^{2}$ is a scale which we have taken to be $m^{2}<<\mu^{2}<<\Lambda^{2}$, it mimics the factorization scale in QCD separating hard and soft dynamics [15]. The above expression is derived using Eqs (3.5), (3.2) and (3.6).

Contribution to $H_{q}\left(x, \Delta^{2}\right)$ comes from one particle and two-particle sectors. The oneparticle sector contributes only when $x=1$ and is a delta function. This receives correction upto order $\alpha$ from the normalization condition of the state. The contribution from the two-particle sector can be written as an overlap of wave functions [18, 22],

$$
\mathcal{N}^{2} \sum \int d^{2} q^{\perp} \psi_{2}^{*}\left(x, q^{\perp}+(1-x) \Delta^{\perp}\right) \psi_{2}\left(x, q^{\perp}\right)
$$

Using the expression Eq. (3.6) we get

$$
\begin{aligned}
H_{q}\left(x, \Delta^{2}\right) & =\mathcal{N}^{2} \delta(1-x) \\
& +\frac{e^{2}}{(2 \pi)^{3}} \mathcal{N}^{2}\left[\int d^{2} q^{\perp} \frac{\frac{1+x^{2}}{1-x}\left(q^{\perp}\right)^{2}+m^{2}(1-x)^{3}}{\left(\left(q^{\perp}\right)^{2}+m^{2}(1-x)^{2}\right)\left(\left(q^{\perp}+(1-x) \Delta^{\perp}\right)^{2}+m^{2}(1-x)^{2}\right)}\right. \\
& \left.+\left(1+x^{2}\right) \int d^{2} q^{\perp} \frac{q^{\perp} \cdot \Delta^{\perp}}{\left(\left(q^{\perp}\right)^{2}+m^{2}(1-x)^{2}\right)\left(\left(q^{\perp}+(1-x) \Delta^{\perp}\right)^{2}+m^{2}(1-x)^{2}\right)}\right] .(3 .
\end{aligned}
$$

One can write this as,

$$
\begin{aligned}
H_{q}\left(x, \Delta^{2}\right)= & \mathcal{N}^{2}\left\{\delta(1-x)+\frac{e^{2}}{(2 \pi)^{3}}\left[( \frac { 1 + x ^ { 2 } } { 1 - x } ) \left[\int \frac{d^{2} q^{\perp}}{L_{2}}\right.\right.\right. \\
& \left.-m^{2}(1-x)^{2} \int \frac{d^{2} q^{\perp}}{L_{1} L_{2}}\right]+m^{2}(1-x)^{3} \int \frac{d^{2} q^{\perp}}{L_{1} L_{2}}+\frac{1+x^{2}}{2(1-x)}\left[\int \frac{d^{2} q^{\perp}}{L_{1}}\right. \\
& \left.\left.\left.-\int \frac{d^{2} q^{\perp}}{L_{2}}-(1-x)^{2}\left(\Delta^{\perp}\right)^{2} \int \frac{d^{2} q^{\perp}}{L_{1} L_{2}}\right]\right]\right\},
\end{aligned}
$$

where $L_{1}=\left(q^{\perp}\right)^{2}+m^{2}(1-x)^{2}$ and $L_{2}=\left(q^{\perp}+(1-x) \Delta^{\perp}\right)^{2}+m^{2}(1-x)^{2}$. In the forward limit using the normalization condition of the state we get,

$$
\begin{aligned}
H_{q}(x, 0)= & \delta(1-x)+\frac{\alpha}{2 \pi}\left\{\left[\frac{1+x^{2}}{1-x} \log \frac{Q^{2}}{\mu^{2}}-\frac{1+x^{2}}{1-x}+1-x\right]\right. \\
& \left.-\delta(1-x)\left[\int d y\left(\frac{1+y^{2}}{1-y} \log \frac{Q^{2}}{\mu^{2}}-\frac{1+y^{2}}{1-y}+1-y\right)\right]\right\} .
\end{aligned}
$$

Integrating over $x$, one gets $\int_{0}^{1} d x H_{q}(x, 0)=F_{1}(0)=1$, where $F_{1}(0)$ is the form factor at zero momentum transfer. Replacing $\alpha$ by $\alpha_{s} C_{f}$ and neglecting the mass, eq. (3.11) reduces to the quark distribution function of a dressed quark and the coefficient of the logarithmic term gives the splitting function $P_{q q}[15]$. At non-zero $\Delta^{\perp}$, in the limit $x \rightarrow 1$, we have

$$
H_{q}\left(x, \Delta^{2}\right) \rightarrow \delta(1-x)+\frac{\alpha}{2 \pi} \log \frac{Q^{2}}{\mu^{2}}\left[\frac{1+x^{2}}{(1-x)_{+}}+\frac{3}{2} \delta(1-x)\right] .
$$


The plus '+' prescription is defined in the usual way. There is no singularity at $x \rightarrow 1$ and $H_{q}\left(x, \Delta^{2}\right)$ is independent of $\Delta^{\perp}$ at $x \rightarrow 1$. The impact parameter dependent parton distribution is obtained by taking a Fourier transform with respect to $\Delta^{\perp}$. At $x \rightarrow 1$, we get

$$
\begin{aligned}
\mathcal{H}_{q}\left(x, b^{\perp}\right)= & \int \frac{d^{2} \Delta^{\perp}}{(2 \pi)^{2}} e^{-i b^{\perp} \cdot \Delta^{\perp}} H_{q}\left(x, \Delta^{2}\right) \\
& =\delta^{2}\left(b^{\perp}\right)\left\{\delta(1-x)+\frac{\alpha}{2 \pi} \log \frac{Q^{2}}{\mu^{2}}\left[\frac{1+x^{2}}{(1-x)_{+}}+\frac{3}{2} \delta(1-x)\right]\right\} .
\end{aligned}
$$

as expected because in this limit the electron carries all the momentum and the transverse width of the impact parameter dependent pdf vanishes [1].

For $x$ not equal to $1, \mathcal{H}_{q}\left(x, b^{\perp}\right)$ has nontrivial $b^{\perp}$ dependency which comes from the (finite) mass terms as well as the $\Delta^{\perp}$ terms. The $q_{\perp}$ integration from which we get the scale dependency in the above expression (Eqn. 3.13) cannot be done analytically for this case. Here we do not plot the scale dependent $\mathcal{H}_{q}\left(x, b^{\perp}\right)$.

Next we consider the helicity flip part of the matrix element :

$$
\begin{gathered}
\int \frac{d y^{-}}{8 \pi} \quad e^{\frac{i}{2} P^{+} y^{-} x}\left\langle P+\Delta, \uparrow\left|\bar{\psi}\left(\frac{-y^{-}}{2}\right) \gamma^{+} \psi\left(\frac{y^{-}}{2}\right)\right| P, \downarrow\right\rangle=\frac{e^{2}}{(2 \pi)^{3}} x(1-x)^{2}(-i m)\left(-i \Delta^{1}-\Delta^{2}\right) \\
\int \frac{d^{2} q^{\perp}}{\left(\left(q^{\perp}\right)^{2}+m^{2}(1-x)^{2}\right)\left(\left(q^{\perp}+(1-x) \Delta^{\perp}\right)^{2}+m^{2}(1-x)^{2}\right)} \\
=-\frac{E_{q}}{2 m}\left(\Delta^{1}-i \Delta^{2}\right) .
\end{gathered}
$$

$m$ is the renormalized mass of the electron. In the limit $\Delta^{\perp}=0$ we get

$$
E_{q}(x, 0)=\frac{\alpha}{\pi} x
$$

Integration over $x$ gives the Schwinger value for the anomalous magnetic moment of an electron in QED :

$$
\int_{0}^{1} E_{q} d x=F_{2}(0)=\frac{\alpha}{2 \pi}
$$

This result can also be obtained by directly calculating the matrix element of the current operator in the light-front framework [14]. 


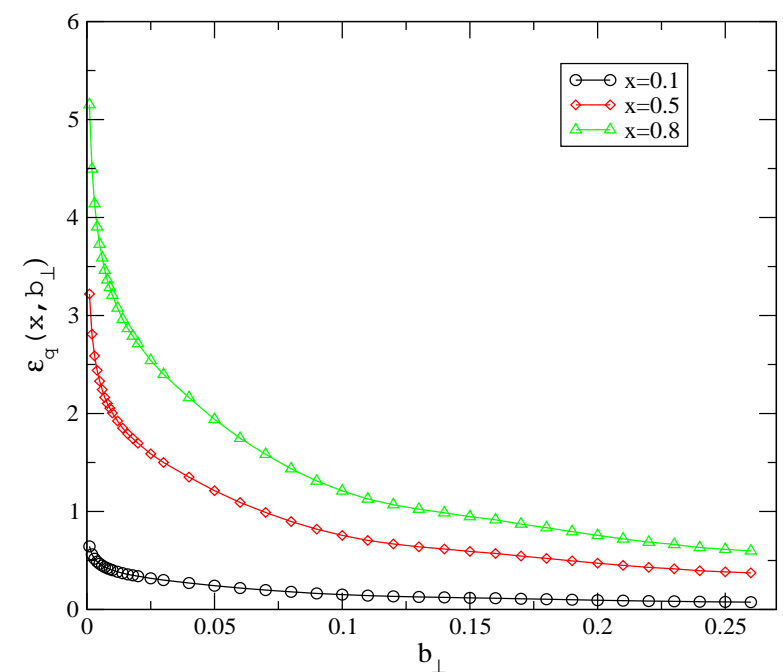

(a)

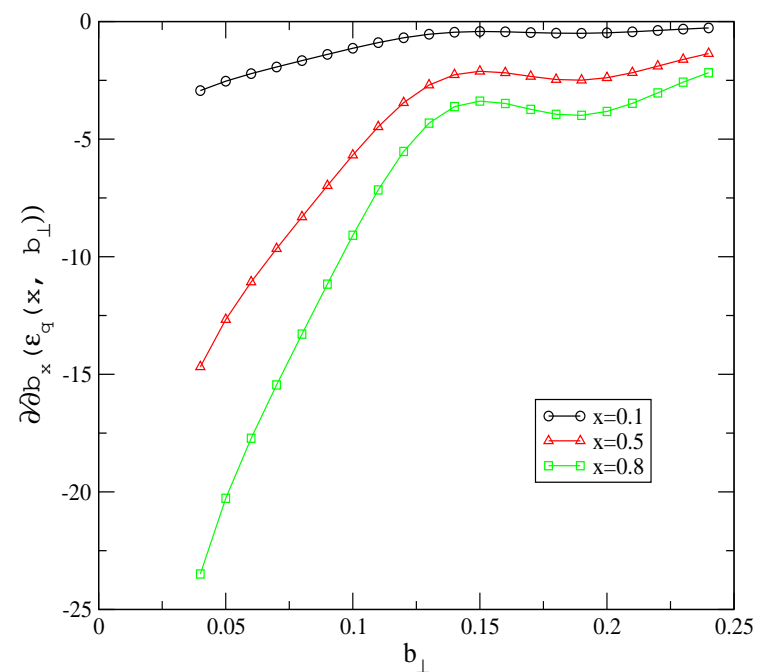

(b)

Fig. 1: (Color online) (a) $\mathcal{E}_{q}\left(x, b^{\perp}\right)$ vs $b^{\perp}$ for three different values of $x$; (b) Derivative of $\mathcal{E}_{q}\left(x, b^{\perp}\right)$ with respect to $b^{x}$ as a function of $b^{\perp}$.

For non-zero $\Delta^{\perp}, E_{q}$ becomes,

$$
E_{q}\left(x, \Delta^{2}\right)=\frac{\alpha}{\pi} m^{2} \int_{0}^{1} d \beta \frac{x}{\left(\beta(1-\beta)\left(\Delta^{\perp}\right)^{2}+m^{2}\right)},
$$

$\beta$ is the Feynman parameter. The scale dependence in this case is suppressed and we can integrate over the full $\left|q^{\perp}\right|$ range, from 0 to $\infty$.

Taking the Fourier transform, we get

$$
\begin{aligned}
\mathcal{E}_{q}\left(x, b^{\perp}\right)= & \int \frac{d^{2} \Delta^{\perp}}{(2 \pi)^{2}} e^{-i b^{\perp} \cdot \Delta^{\perp}} E_{q}\left(x,-\left(\Delta^{\perp}\right)^{2}\right) \\
& =\int \frac{\Delta d \Delta}{(2 \pi)^{2}} d \theta E_{q}\left(x,-\Delta^{2}\right) e^{-i b \Delta \cos \theta} \\
& =\frac{1}{2 \pi} \int_{0}^{\infty} \Delta d \Delta E_{q}\left(x,-\Delta^{2}\right) J_{0}(b \Delta) .
\end{aligned}
$$

For numerical calculation we use the real integral form of the Bessel function :

$$
J_{0}(b \Delta)=\frac{1}{\pi} \int_{0}^{\pi} \cos (b \Delta \sin \theta) d \theta .
$$

If we take a state polarized in the $\hat{y}$ direction (in the infinite momentum frame) and whose center of momentum is at the origin,

$$
\left|P^{+}, R^{\perp}=0, \hat{y}\right\rangle=\frac{1}{\sqrt{2}}\left(\left|P^{+}, R^{\perp}=0, \uparrow\right\rangle+i\left|P^{+}, R^{\perp}=0, \downarrow\right\rangle\right) ;
$$


the unpolarized fermion distribution in the impact parameter space gets distorted,

$$
\begin{aligned}
q_{\hat{y}}\left(x, b^{\perp}\right)= & \left\langle P^{+}, R^{\perp}=0^{\perp}, \hat{y}\left|O_{q}\left(x, b^{\perp}\right)\right| P^{+}, R^{\perp}=0^{\perp}, \hat{y}\right\rangle \\
& =\mathcal{H}_{q}\left(x, b^{\perp}\right)+\frac{1}{2 m} \frac{\partial}{\partial b^{x}} \mathcal{E}_{q}\left(x, b^{\perp}\right) .
\end{aligned}
$$

The distortion is directly related to $\mathcal{E}_{q}\left(x, b_{\perp}\right)$. Fig. 1 (a) shows the helicity flip contribution $\mathcal{E}_{q}\left(x, b^{\perp}\right)$ as a function of $b^{\perp}$ for three different values of $x$. We have plotted for positive $b^{\perp} . \mathcal{E}_{q}\left(x, b^{\perp}\right)$ is a smooth function of $b^{\perp}$ in the range shown and it increases as $b^{\perp}$ decreases. Also, it increases linearly with $x$, as is clear from Eq. (3.17). We have taken the overall normalization $\frac{\alpha}{2 \pi}=1$ in order to study the qualitative behavior and $m=0.5 . \mathcal{E}_{q}\left(x, b^{\perp}\right)$ has a maximum at $b^{\perp}=0$. Fig. 1 (b) shows the derivative of $\mathcal{E}_{q}\left(x, b^{\perp}\right)$ with respect to $b^{x}$, which gives the distortion of the pdf of an electron at one loop in impact parameter space due to transverse polarization. The integrand in this case contains $J_{1}(b \Delta)$ and it is highly oscillatory. However, the integral converges for the $b^{\perp}$ range shown in the plot. As $\mathcal{E}_{q}\left(x, b^{\perp}\right)$ is a smooth function with maximum at $b^{\perp}=0$ its derivative for positive $b^{\perp}$ is negative. One can see that the distortion of the distribution in impact parameter space increases as $b^{\perp}$ decreases and for a given $b^{\perp}$ the distortion is higher in magnitude for larger values of $x$. The distortion shifts the distribution actually toward negative values of $b^{\perp}$.

\section{GAUGE BOSON DISTRIBUTIONS}

From the definition,

$$
H_{g}\left(x, \Delta^{2}\right)=\frac{1}{8 \pi x P^{+}} \int d y^{-} e^{\frac{i}{2} P^{+} y^{-} x}\left\langle P^{\prime}, \uparrow\left|F^{+\nu}\left(-\frac{y^{-}}{2}\right) F_{\nu}^{+}\left(\frac{y^{-}}{2}\right)\right| P, \uparrow\right\rangle
$$

we take the state to be a dressed electron as in Eq. (3.2). Contribution in this case comes only from the two-particle sector, and we get

$$
\begin{aligned}
H_{g}\left(x, \Delta^{2}\right)= & \mathcal{N}^{2} \int d^{2} q^{\perp} \psi_{2}^{*}\left(1-x, q^{\perp}\right) \psi_{2}\left(1-x, q^{\perp}+(1-x) \Delta^{\perp}\right) \\
= & \frac{\alpha}{(2 \pi)^{2}}\left\{\frac{\left(1+(1-x)^{2}\right)}{x} \int \frac{d^{2} q^{\perp}}{L_{2}}+m^{2} x^{3} \int \frac{d^{2} q^{\perp}}{L_{1} L_{2}}+\frac{1+(1-x)^{2}}{2 x}\left[\int \frac{d^{2} q^{\perp}}{L_{1}}\right.\right. \\
& \left.\left.-\int \frac{d^{2} q^{\perp}}{L_{2}}-(1-x)^{2}\left(\Delta^{\perp}\right)^{2} \int \frac{d^{2} q^{\perp}}{L_{1} L_{2}}\right]\right\}
\end{aligned}
$$

where $L_{1}=\left(q^{\perp}\right)^{2}+m^{2} x^{2}$ and $L_{2}=\left(q^{\perp}+(1-x) \Delta^{\perp}\right)^{2}+m^{2} x^{2}$. The scale dependency as before, comes from the limits of the $q^{\perp}$ integrations. Fourier transform of the above expression gives 


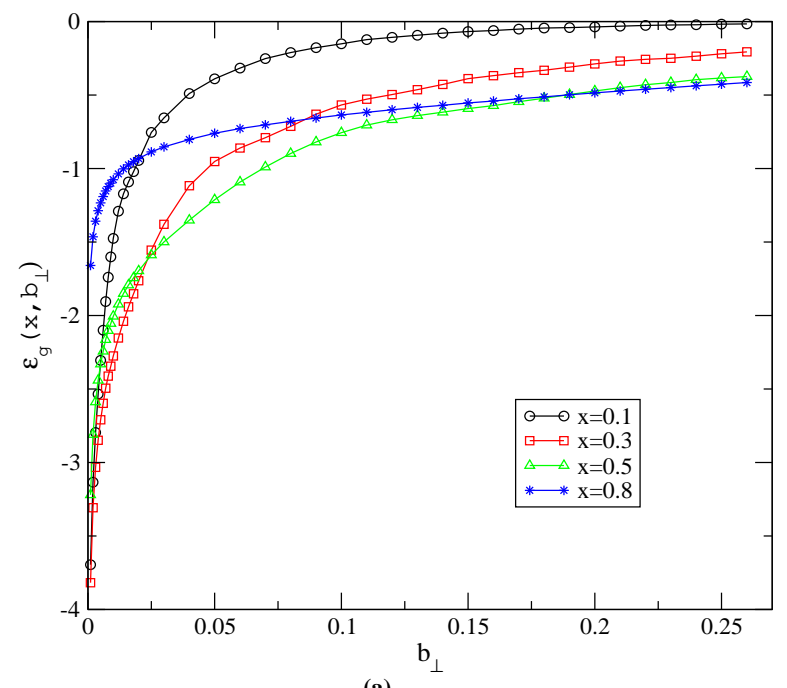

(a)

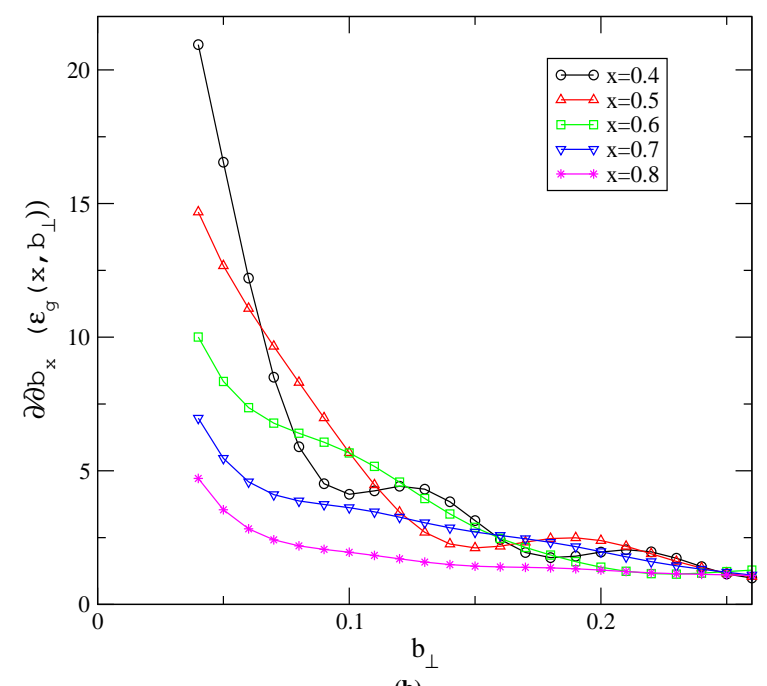

(b)

Fig. 2: (Color online) (a) $\mathcal{E}_{g}\left(x, b^{\perp}\right)$ vs $b^{\perp}$ for four different values of $x$; (b) Derivative of $\mathcal{E}_{g}\left(x, b^{\perp}\right)$ with respect to $b^{x}$ as a function of $b^{\perp}$.

the impact parameter dependent gauge boson pdf. Nontrivial $b^{\perp}$ dependence come from the mass term and the $\Delta^{\perp}$ term. The other terms give logarithmic dependence on the scale. In the forward limit,

$$
H_{g}(x, 0)=\frac{\alpha}{2 \pi}\left[\frac{1+(1-x)^{2}}{x}\right] \log \frac{Q^{2}}{\mu^{2}}
$$

Next we look at the helicity flip part of the matrix element. This can be written as,

$$
\int \frac{d y^{-}}{8 \pi} e^{\frac{i}{2} P^{+} y^{-} x}\left\langle P+\Delta, \uparrow\left|F^{+\nu}\left(\frac{-y^{-}}{2}\right) F_{\nu}^{+}\left(\frac{y^{-}}{2}\right)\right| P, \downarrow\right\rangle=-\frac{E_{g}}{2 m}\left(\Delta^{1}-i \Delta^{2}\right),
$$

which gives

$$
E_{g}\left(1-x, \Delta^{2}\right)=-\frac{\alpha}{\pi} m^{2} \int_{0}^{1} d \beta \frac{x(1-x)^{2}}{\left(\beta(1-\beta)\left(\Delta^{\perp}\right)^{2}(1-x)^{2}+m^{2} x^{2}\right)} .
$$

If we denote the momentum fraction of the quark as $x$ instead of $1-x$, we get,

$$
E_{g}\left(x, \Delta^{2}\right)=-\frac{\alpha}{\pi} m^{2} \int_{0}^{1} d \beta \frac{x^{2}(1-x)}{\left(\beta(1-\beta)\left(\Delta^{\perp}\right)^{2} x^{2}+m^{2}(1-x)^{2}\right)} .
$$

In the forward limit, this gives,

$$
E_{g}(x, 0)=-\frac{\alpha}{\pi} \frac{x^{2}}{1-x}
$$


The second moment of $E_{q, g}(x, 0), \int d x x E_{q, g}(x, 0)$ gives in units of $\frac{1}{2 m}$ by how much the transverse center of momentum of the parton $q, g$ is shifted away from the origin in the transversely polarized state. When summed over all partons, the transverse center of momentum would still be at the origin. Indeed it is easy to check for a dressed electron

$$
\int_{0}^{1} d x x E_{q}(x, 0)+\int_{0}^{1} d x(1-x) E_{g}(x, 0)=0,
$$

which is due to the fact that the anomalous gravitomagnetic moment of the electron has to vanish. Note that in the second term, $(1-x)$ is the momentum fraction of the gauge boson. In fact the second moment of $E_{q, g}$ appear in the angular momentum sum rule [20] and they are related to the orbital angular momentum of the nucleon. In the approach we are following, this connection can be seen from the fact that in the light-front gauge, the matrix element of the quark orbital angular momentum operator cancels the contribution of the gluon helicity and orbital angular momentum for a dressed quark in the helicity sum rule [16].

The impact parameter dependent gauge boson pdf can be defined in the same way as in Eq. (3.18):

$$
\begin{aligned}
\mathcal{E}_{g}\left(x, b^{\perp}\right)= & \int \frac{d^{2} \Delta^{\perp}}{(2 \pi)^{2}} e^{-i b^{\perp} \cdot \Delta^{\perp}} E_{g}\left(x,-\left(\Delta^{\perp}\right)^{2}\right) \\
& =\int \frac{\Delta d \Delta}{(2 \pi)^{2}} d \theta E_{g}\left(x,-\Delta^{2}\right) e^{-i b \Delta \cos \theta} \\
& =\frac{1}{2 \pi} \int_{0}^{\infty} \Delta d \Delta E_{g}\left(x,-\Delta^{2}\right) J_{0}(b \Delta) .
\end{aligned}
$$

Fig. 2 (a) shows $\mathcal{E}_{g}\left(x, b^{\perp}\right)$ vs $b^{\perp}$ for three different values of $x . \mathcal{E}_{g}\left(x, b^{\perp}\right)$ is negative for positive $b^{\perp}$ and has a negative maximum at $b^{\perp}=0$. As before, we took $\frac{\alpha}{2 \pi}=1$ and $m=0.5$. Fig. 2 (b) shows the derivative of $\mathcal{E}_{g}\left(x, b^{\perp}\right)$ with respect to $b^{x}$ as a function of $b^{\perp}$. Fig. 3 (a) shows $\mathcal{E}_{g}\left(x, b^{\perp}\right)$ as a function of $x$ for three different values of $b_{\perp}$. Unlike the fermion case, $\mathcal{E}_{g}\left(x, b^{\perp}\right)$ for a fixed $b^{\perp}$ is a complicated function of $x$ and does not increase monotonically as $x$ increases. Depending on $b^{\perp}$, the maximum of $\mathcal{E}_{g}\left(x, b^{\perp}\right)$ appears for different $x(0 \leq x \leq 1)$. Again in contrary to the fermionic case, $\mathcal{E}_{g}\left(x, b^{\perp}\right)$ becomes smoother when $x$ is large which is expected since for large $x(x=0.7,0.8$ in Fig. $2(\mathrm{~b}))$, gauge boson carries only a small $(1-x)$ fraction of total momentum. Fig. $3(\mathrm{~b})$ shows the derivative of $\mathcal{E}_{g}\left(x, b^{\perp}\right)$ with respect to $b^{x}$ as a function of $x$, which shows the distortion of the pdf in the transverse plane for a transversely polarized state. 


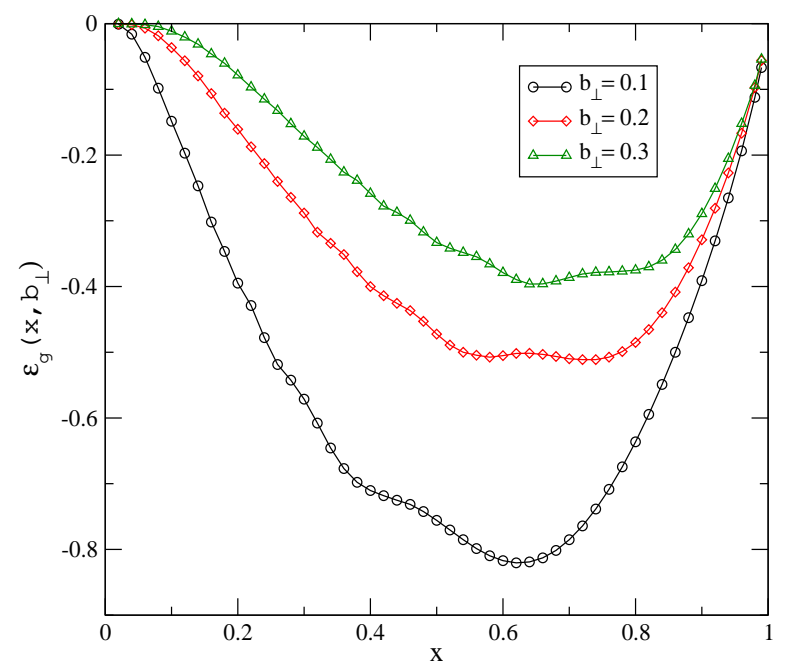

(a)

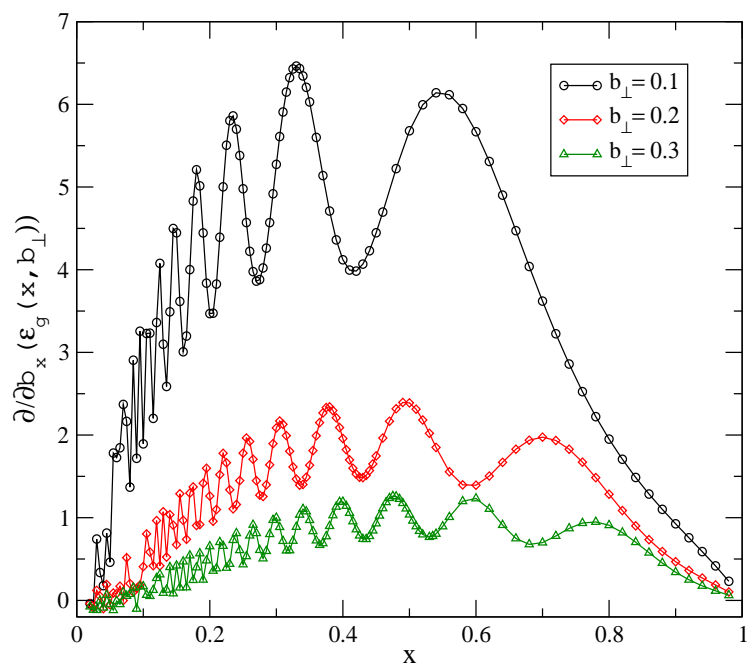

(b)

Fig. 3: (Color online) (a) $\mathcal{E}_{g}\left(x, b^{\perp}\right)$ vs $x$ for three different values of $b_{\perp}$; (b) Derivative of $\mathcal{E}_{g}\left(x, b^{\perp}\right)$ with respect to $b_{x}$ as a function of $x$.

\section{SUMMARY}

We investigated the impact parameter dependent parton distributions for a relativistic composite system. An ideal framework is based on light-front field theory, where the transverse boosts behave like Galilean boosts and the longitudinal boost operator produces just a scale transformation. We take an effective composite spin $1 / 2$ state, namely an electron dressed with a photon in QED. Using the overlap representation of GPDs in terms of lightcone wave functions, we obtained the scale dependence of the impact parameter dependent pdfs at one loop. We also showed the distortion of the fermion and gauge boson distributions in the transverse plane when the state is transversely polarized. Plots show the qualitative behaviors of the helicity flip contributions $\mathcal{E}_{q}\left(x, b^{\perp}\right)$ and $\mathcal{E}_{g}\left(x, b^{\perp}\right)$ respectively for the electron and the gauge boson and their derivatives with respect to $b_{x}$ which give the distortion of the distribution in the transverse plane. For both fermion and gauge boson, for a fixed $x$, the distortions are larger in magnitude for smaller $b_{\perp}$.

\section{ACKNOWLEDGMENT}

We thank M. Burkardt for valuable discussions and S.J. Brodsky for interesting comments. We also thank the organisers of WHEPP-8 at IIT Bombay where part of this work was carried 
out. The work of AM has been supported in part by the 'Bundesministerium für Bildung und Forschung', Berlin/Bonn and the work of DC was partially suported by the Department of Energy under Grant No. DE-FG02-97ER-41029.

[1] M. Burkardt, Int. Jour. Mod. Phys. A 18, 173 (2003).

[2] M. Burkardt, Phys. Rev. D 62, 071503 (2000), Erratum- ibid, D 66, 119903 (2002); J. P. Ralston and B. Pire, Phys. Rev. D 66, 111501 (2002).

[3] For reviews on generalized parton distributions, see M. Diehl, Phys. Rept, 388, 41 (2003); X. Ji, J. Phys. G 24, 1181 (1998); A. V. Radyushkin, hep-ph/0101225, published in "At the Frontier of Particle Physics/Handbook of QCD", ed. M. Shifman (World Scientific, Singapore, 2001); K. Goeke, M. V. Polyakov, M. Vanderhaeghen, Prog. Part. Nucl. Phys. 47, 401 (2001).

[4] D. W. Sivers, Phys. Rev. D 43, 261 (1991).

[5] M. Burkardt and D. S. Hwang, Phys. Rev. D 69, 074032 (2004).

[6] W. Bronioski, E. R. Arriola, Phys. Lett. B 574, 57 (2003).

[7] S. Scopetta, V. Vento, Phys. Rev. D 69, 094004 (2004).

[8] A. Mukherjee, I. V. Musatov, H. C. Pauli and A. V. Radyushkin, Phys. Rev D 67, 073014 (2003).

[9] S. Liuti, S. K. Taneja, hep-ph/0405014.

[10] S. Dalley, Phys. Lett. B 570, 191 (2003).

[11] LHPC and SESAM collaboration, hep-lat/0312014.

[12] K. G. Wilson, T. S. Walhout, A. Harindranath, W. M. Zhang, R. J. Perry and S. D. Glazek, Phys. Rev. D 49, 6720 (1994).

[13] D. Chakrabarti and A. Mukherjee, hep-ph/0410126.

[14] S. J. Brodsky, D. S. Hwang, B-Q. Ma, I Schmidt, Nucl. Phys. B 593, 311 (2001).

[15] A. Harindranath, R. Kundu, W. M. Zhang, Phys. Rev D 59, 094013; A. Harindranath, R. Kundu, A. Mukherjee, J. P. Vary, Phys. Rev. D 58, 114022 (1998), A. Mukherjee and D. Chakrabarti, Phys. Lett. B 506, 283 (2001).

[16] A. Harindranath and R. Kundu, Phys. Rev. D 59, 116013 (1999)

[17] A. Harindranath, A. Mukherjee, R. Ratabole, Phys. Lett. B 476, 471 (2000); Phys. Rev. D 63, $045006(2001)$. 
[18] A. Mukherjee and M. Vanderhaeghen, Phys. Rev. D 67, 085020 (2003), Phys. Lett. B 542, $245(2002)$.

[19] M. Diehl, Eur. Phys. Jour. C 25, 223 (2002).

[20] X. Ji, Phys. Rev. Lett. 78, 610 (1997); Phys. Rev. D55, 7114 (1997).

[21] A. Harindranath, An Introduction to the Light Front Dynamics for Pedestrians in Lightfront Quantization and Non-perturbative QCD , Ed. J. P. Vary and F. Wolz, published by Internatinal Institute of Theoretical and Applied Physics, Ames, Iowa, USA (1997).

[22] M. Diehl, T. Feldmann, R. Jacob, P. Kroll, Nucl. Phys. B 596, 33 (2001), Erratum-ibid 605, 647 (2001). 